P-65 MENSTRUAL DISORDERS IN HAIRDRESSERS: A CROSSSECTIONAL STUDY

${ }^{1}$ Aouatef Mahfoudh, Raja Ammar, Asma Kheder, Noura Bel Haj, Amira Omrane, Taoufik Khalfallah. 'University of Monastir, Tunisia

\subsection{6/OEM-2021-EPI. 184}

Aims Determine the frequency of menstrual disorders and identify their risk factors in hairdressers of childbearing age.

Methods In a cross-sectional study, participants were all female hairdressers below 50 years of age working in the region of Mahdia in Tunisia $(n=80)$. A control group comprised female office workers $(n=80)$, with respective matching criteria. Menstrual disorders were defined as short cycles, long cycles, irregular cycles and bleeding or spotting between periods (intermenstrual bleeding). The data were analyzed by logistic regression calculating odds ratios (OR).

Results The frequency of menstrual disorders was significantly more frequent in the hairdressing population with a predominance of irregular menstrual cycle type disorders (51.3\%), followed by short cycle type disorders (20\%). Menstrual disorders were significantly associated with occupation $(\mathrm{p}=$ $0.00)$, non-use of gloves $(p=0.00)$, ventilation $(p=0.00)$ and the presence of a closed waste cycle $(p=0.00)$. After applying logistic regression, hairdressers were significantly more at risk of menstrual disorders $(p=0.001)$ than female office workers with an $\mathrm{OR}=4.31$ (95\% CI $=1.8-10.33$ ).

Conclusion A disruption of the menstrual cycle can interfere with the fertility of workers and be the cause of delayed contraception. This is why preventive actions at different scales need to be applied.

\section{P-68 OCCUPATIONAL HEALTH AND SAFETY PRACTICES IN A SMALL CONSTRUCTION COMPANY IN PIURA, PERU}

${ }^{1}$ Norvil Mera Chu. 'Universidad de Piura, Peru

\subsection{6/OEM-2021-EPI. 185}

Introduction Participatory programs for occupational risk management are becoming more important in small workplaces in developing countries such as Peru. The current legislation on occupational health and safety (OHS) in Peru is more focused on occupational exposure in big enterprises, however. In small construction companies, workers have significant high-risk occupational exposure to suffer accidents and diseases related to physical, locative, ergonomic and mechanical factors. Most of these companies do not have an OHS system adapted to their reality.

Objectives The purpose was to describe the implementation of the OHS System and its progressive improvement during 3 years in a small construction company in Peru and to show how it turned out to be a good investment and a competitive advantage.

Methods This research was a case report study. The unit of study was the economic indicators of expenses and income of a small construction company, related to OHS activities as training programs, involving workers in safety inspections, performing medical examinations for workers, performing internal audits, among others. Besides, the impact of the system on the frequency of accidents and illnesses among workers, on absenteeism, on labour inspections, on getting clients, etc. was analyzed. The instrument used was a data collection sheet. A database was created in Microsoft Excel 2007. SPSS software package was used.

Results After 3 years since the OHS system implementation, the frequency of accidents was reduced by $25 \%$. Absenteeism was reduced by $30 \%$. The enterprise got its OHS system certified and got more clients due to its OHS standards, which implied more economic income and a worth difference compared to other companies.

Conclusion It was a good investment to implement and improve the OHS system. It is necessary to implement studies in other small enterprises in developing countries.

\section{P-69 TEACHING INTERVENTIONS IN OCCUPATIONAL HEALTH AND SAFETY SYSTEM IN A SMALL METAL MECHANIC COMPANY IN PIURA, PERU}

${ }^{1}$ Norvil Mera Chu. ${ }^{1}$ Universidad de Piura, Peru

\subsection{6/OEM-2021-EPI.186}

Introduction There are several small enterprises in Peru that provide services to mining, construction and oil companies which increase the probability of accidents and diseases. Interventions to educate and train workers in prevention, especially participatory programs for occupational risk reduction, are becoming very important in small workplaces in developing countries like Peru, where there is a significant work population which is underserved and includes people with some issues such as low-literacy and limited professional academic training.

Objective The purpose of the present study was to describe the implementation of teaching interventions and its progressive improvement during 3 years in a small metal mechanic company in Peru and to show its impact in the prevention culture.

Methods The unit of this case report study was the indicators of teaching interventions as number of participants, professions, time working in Occupational Health and Safety (OHS), education methods used and a survey at the end of intervention. Besides, the impact of the intervention on the frequency of accidents and illnesses in workers, on absenteeism and on labor inspections was analyzed. The instrument used was a data collection sheet.

Results During 3 years, the teaching interventions implemented were case discussions, role games and performance-feedback. The frequency of accidents was reduced by $22 \%$. Absenteeism was reduced by $33 \%$.

Conclusion Teaching interventions had goods results in prevention culture by reducing accidents and absenteeism in this small metal mechanic company. It is necessary to implement studies for assessing the effect of teaching interventions in OHS in other small enterprises in developing countries.

\section{P-75 CORRELATES OF POST-TRAUMATIC STRESS DISORDER (PTSD) AMONG AMBULANCE PERSONNEL IN THE WESTERN CAPE PROVINCE, SOUTH AFRICA}

${ }^{1}$ Itumeleng Ntatamala, Shahieda Adams. 'University of Cape Town, South Africa

\subsection{6/OEM-2021-EPI.187}

Introduction Ambulance personnel are exposed to various occupational stressors which predispose to the development 